\title{
Contour Tracking Control Based on Extended State Observer for Multiaxis Motion System
}

\author{
Sanxiu Wang $\mathbb{D}^{\mathrm{D}}$, Qiang Zhou, and Yang Wang \\ College of Electronic and Information Engineering, Taizhou University, Taizhou, Zhejiang, 318000, China \\ Correspondence should be addressed to Sanxiu Wang; wsx8188@163.com
}

Received 10 December 2019; Revised 16 February 2020; Accepted 28 February 2020; Published 29 March 2020

Academic Editor: Javier Moreno-Valenzuela

Copyright ( $\odot 2020$ Sanxiu Wang et al. This is an open access article distributed under the Creative Commons Attribution License, which permits unrestricted use, distribution, and reproduction in any medium, provided the original work is properly cited.

In the contouring process, the trajectory generated by the computer numerical control (CNC) machine tool is a result of the multiaxis coordinated motion. The contour error has a direct impact on the accuracy of the machined product. To obtain higher contouring accuracy of the multiaxis motion control system, this paper presents a cross-coupled control approach based on the extended state observer sliding mode control. First, a single-axis sliding mode controller is designed, and an extended state observer is used to estimate system disturbances and improve the system robustness. Then, the cross-coupled control approach handles the coordinated motion of multiple axes to improve the contour control accuracy. Next, a simulation study is conducted on the three-axis motion platform. Its result shows that the control algorithm is effective in reducing tracking errors and contour errors.

\section{Introduction}

With the rapid development of the modern industry, there is an increasing demand on higher accuracy for the multiaxis manufacturing systems. To improve the contouring accuracy, many experts and scholars are devoted to researching various single-axis tracking control strategies, thereby improving the contour motion control accuracy indirectly [1-3]. However, this conventional contour controller based on the single-axis uncoupled control cannot fundamentally solve the problem of contour control. For example, when an axis has a large error under disturbances, the other axes do not take necessary measures to reduce the impact of the performance degradation of the axis on the overall performance because they still consider that the axis works normally. Therefore, to solve the shortcomings of such a single-axis uncoupled controller, it is necessary to introduce a coupled mechanism among multiple axes, with the contour error as the control target, to directly reduce the contour error and improve the contouring accuracy.

Karen proposed the cross-coupled control (CCC) to solve the contour motion problem of the two-axis feed system. On this basis, variable gain CCC is further proposed
$[4,5]$. Since then, more research studies on optimized CCC have been reported [6-10]. In CCC, the multiaxis control system is considered as organic whole where internal components are interconnected. The error compensation is calculated using the relations between the axes and fed back to each single axis for correction, thereby improving the matching and coordination between the axes. By changing the open-loop to the closed-loop contour control, CCC achieves the purpose of contour control and improves the control accuracy of the system.

The development of the contour control technology results in increased complexity of the cross-coupled controller enhanced robust stability, and better coordination, but the anti-interference capacity is less improved. The multiaxis motion platform is susceptible to external disturbances in the machining process, which affects the position accuracy and contour accuracy of the CNC machining system. Therefore, this paper introduces an extended state observer (ESO) to estimate the disturbances and combines it with the sliding mode controller (SMC) to improve the position tracking accuracy of the single axis. Moreover, the cross-coupled controller is used to deal with the coordinated motion among the axes to ensure the accuracy of the contour 
control. The simulation study on the three-axis motion platform demonstrates that the approach can effectively reduce the impact of external disturbances, improve the coordination among the axes, and ensure the tracking accuracy and contour accuracy.

\section{Contour Error Model}

Mismatch of dynamic characteristics of each axis motor is the main cause of contour error [11]. This paper applies CCC to coordinate the motion of each axis. The contour error is calculated by selecting the appropriate CCC gain. Then, the contour error compensation is assigned to each axis, respectively. Therefore, it is critical to build a more accurate contour error model. For the noncircular arbitrary contour, literature [12] proposes an effective two-axis contour error vector estimation approach and further introduces the multiaxis contour error estimation.

The diagram of 3-axis motion contour error vectors is shown in Figure 1.

$P$ and $R$ are actual position and reference position, respectively, $e$ represents the tracking error between the reference position and the actual position, $\varepsilon$ is the contour error, for the deviation between the actual position and the desired contour, and $t$ is the normalized tangential vector. Because it is difficult to calculate the precise value of contour error in practice, the estimation of contour error $\widehat{\varepsilon}$ is adopted. Contour error estimation $\widehat{\varepsilon}$ depends on tracking error $e$ and normalize tangential vector $t$; when tracking error $\|e\|$ is small enough, the contour error $\varepsilon$ can be closely approximated by the contour error estimation vector $\widehat{\varepsilon}$.

Define the normalized estimated contouring error vector $n$ :

$$
n=\eta_{1} t+\eta_{2} e
$$

which satisfies the following condition:

$$
\begin{gathered}
\langle n, t\rangle=0, \\
\|n\|=1, \\
\|t\|=1 .
\end{gathered}
$$

The relation between $\eta_{1}$ and $\eta_{2}$ can be derived from the above condition:

$$
\eta_{1}=-\eta_{2} \cdot\langle e, t\rangle
$$

Combining formulas (3) and (5), according to the properties of the vector inner product, the following are obtained:

$$
\begin{aligned}
& \eta_{1}=\mp \frac{\langle e, t\rangle}{\sqrt{\|e\|^{2}-\langle e, t\rangle^{2}}} \\
& \eta_{2}= \pm \frac{1}{\sqrt{\|e\|^{2}-\langle e, t\rangle^{2}}}
\end{aligned}
$$

in which the signs of $\eta_{1}$ and $\eta_{2}$ determine the direction of the normalized estimation contour error vector. Because the

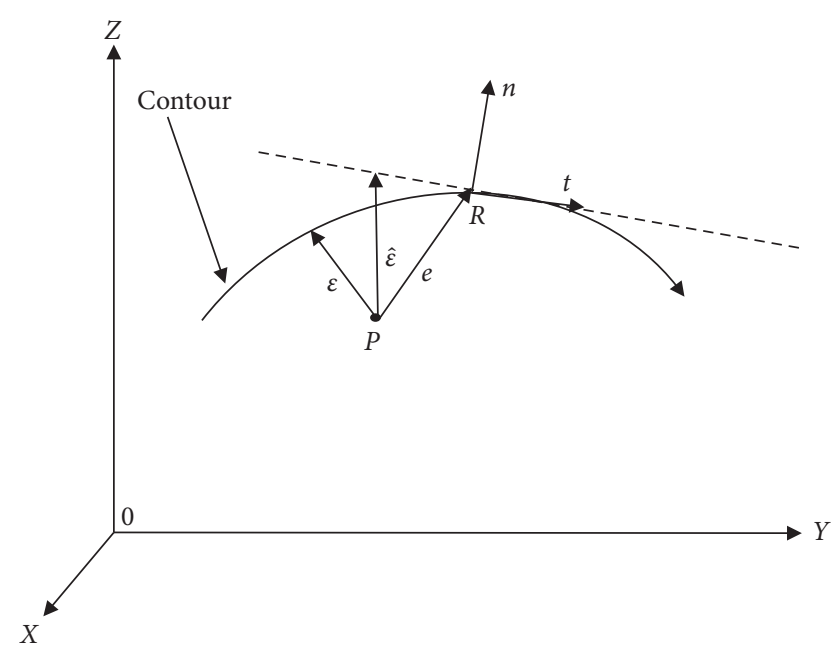

Figure 1: Contour error vector graph of 3-axis motion control systems.

angle between the normalized estimated contour error and the tracking error is within the range $\left[-90^{\circ}, 90^{\circ}\right]$ and satisfies $\langle n, e\rangle \geq 0, \eta_{1}$ and $\eta_{2}$ can be further determined as

$$
\begin{aligned}
& \eta_{1}=-\frac{\langle e, t\rangle}{\sqrt{\|e\|^{2}-\langle e, t\rangle^{2}}}, \\
& \eta_{2}=-\frac{1}{\sqrt{\|e\|^{2}-\langle e, t\rangle^{2}}} .
\end{aligned}
$$

It can be seen from Figure 1 and equation (1) that the estimated contour error vector $\widehat{\varepsilon}$ is the inner product of the normalized estimated contouring error vector $n$ and the tracking error vector $e$, that is,

$$
\|\widehat{\varepsilon}\|=\langle n, e\rangle .
$$

Therefore, the estimated contour error vector is

$$
\widehat{\varepsilon}=\|\widehat{\varepsilon}\| \cdot n=\langle n, e\rangle \cdot n .
$$

The magnitude of the estimated contour error vector can be regulated by the cross-coupled controller and compensated for each axis along the direction of the estimated contour error vector, wherein how much compensation is made for each axis is determined by the cross-coupled gain. Therefore, the cross-coupled gain can be obtained directly from the normalized estimated contour error vector.

Let $n=\left[n_{x}, n_{y}, n_{z}\right]^{T}$, and CCC gain $C=\left[C_{x}, C_{y}, C_{z}\right]^{T}$ can be expressed directly as

$$
\begin{aligned}
C_{i} & =n_{i}, \\
i & =x, y, z .
\end{aligned}
$$

The contour error is

$$
\varepsilon=C_{x} e_{x}+C_{y} e_{y}+C_{z} e_{z}
$$

Since this approach is efficient for coupling calculation of nonlinear contours, it is used to estimate the contour error coupled gain in the cross-coupled controller in this paper. 


\section{System Model}

Of all permanent magnet synchronous motors (PMSM), the permanent magnet linear synchronous motor (PMLSM) is a special type of motor in which the stator is arranged in a straight line while the mover moves linearly along the stator direction. Driven by the electromagnetic force, the linear motor provides direct linear motion with the absence of a mechanical transmission mechanism, thus eliminating the additional mechanical losses. It features high efficiency and high motion control accuracy, which are ideal for the applications with linear motion control characteristics, such as precision machine tools and automated production lines.

PMLSMs which are perpendicular to each other are used to contour control the three-axis motion platform in this paper. The mechanical motion equation is

$$
F_{e}=K_{f} i_{q}=M \dot{v}+B v+d,
$$

where $F_{e}$ is the electromagnetic thrust, $K_{f}$ is the electromagnetic thrust coefficient, $M$ is the total mass of the mover and the load carried by the mover, $B$ is the viscous friction coefficient, $v$ is the speed of the motor mover, $\dot{v}$ is the acceleration of the motor mover, and $d$ is the external disturbance.

The motor position $q$ and the motor speed $v$ are selected as system state variables, and the state equation of PMLSM can be written as

$$
\left\{\begin{array}{l}
\dot{q}=v, \\
\dot{v}=-\frac{B}{M} \dot{q}+\frac{K_{f}}{M} u+d .
\end{array}\right.
$$

$u=i_{q}$ is the motor control input. The direct drive three-axis motion platform system model can be represented by three second-order differential equations:

$$
\left\{\begin{array}{l}
\ddot{q}_{1}=-\frac{B_{1}}{M_{1}} \dot{q}_{1}+\frac{K_{f 1}}{M_{1}} u_{1}+d_{1}, \\
\ddot{q}_{2}=-\frac{B_{2}}{M_{2}} \dot{q}_{2}+\frac{K_{f 2}}{M_{2}} u_{2}+d_{2}, \\
\ddot{q}_{3}=-\frac{B_{3}}{M_{3}} \dot{q}_{3}+\frac{K_{f 3}}{M_{3}} u_{3}+d_{3} .
\end{array}\right.
$$

\section{Controller Design}

In the actual machining process, the multiaxis motion control systems generally have uncertain nonlinearities, strong coupling, and external disturbances, which affect the position accuracy and contour accuracy of the CNC machining systems. Therefore, to meet the machining accuracy requirement of the three-axis motion platform, this paper designs the controller from two aspects: single-axis position control and contour control. For the single-axis motion, an ESO-based sliding mode controller is used to improve single-axis position tracking accuracy and single-axis robustness is ensured. For the motion between axes, the CCC algorithm is used to improve contour control accuracy. The proposed contour error CCC scheme is shown in Figure 2.

$q_{x d}, q_{y d}$, and $q_{z d}$ are the reference input position of $x$, $y$, and $z$ axes, respectively, and $q_{x}, q_{y}$, and $q_{z}$ are actual output position of three axes. $\varepsilon$ is the contour error. $P_{x}, P_{y}$, and $P_{z}$ represent the controlled object of three axes.

The workflow is described as follows:

(1) Three-axis tracking error $e_{x}, e_{y}$, and $e_{z}$ is obtained by the reference input and the actual output of each axis

(2) Three-axis tracking errors are combined with the contour error gains $C_{x}, C_{y}$, and $C_{z}$ to obtain the contour error $\varepsilon$

(3) Three-axis tracking errors are processed with the sliding mode controllers of $x, y$, and $z$ axes, respectively, to obtain the control signals $u_{x}, u_{y}$, and $u_{z}$

(4) After processed by the cross-coupled controller, the contour error is combined with $C_{x}, C_{y}$, and $C_{z}$ to obtain the contour error compensation of each axis

(5) By taking the sliding mode control amount and the actual output of each axis as the input signals of the ESO, the observations of the position signals, speed signals, and disturbance signals of the respective axis are generated and fed back to the SMC

(6) $u_{x}, u_{y}$, and $u_{z}$ and the contour error compensation of each axis are superimposed as the total control amount which is applied on each axis actuator with perturbation; thus, the contour motion control is achieved.

4.1. Extended State Observer Design. The single-axis motion control is susceptible to disturbance, thereby degrading the control performance. To solve this problem, an ESO-based disturbance compensation approach is proposed in this section. The basic idea for ESO is to take the disturbance quantity in the system as a state quantity and combine it with the original state variables to construct the extended state observations; then, we can use a few measurable data in the system model to estimate the external disturbances and system state information that cannot be measured. As a result, the impact of disturbances on the system can be traded off to improve the system's antidisturbance and robustness $[13,14]$.

Assume the state variables $x_{1}=q$ and $x_{2}=v=\dot{q}$, and system (15) can be written as the following state equation:

$$
\left\{\begin{array}{l}
\dot{x}=A x+G\left(\frac{K_{f}}{M} u+d\right), \\
y=C x,
\end{array}\right.
$$

in which $x=\left[\begin{array}{l}x_{1} \\ x_{2}\end{array}\right]=\left[\begin{array}{l}q \\ \dot{q}\end{array}\right], A=\left[\begin{array}{cc}0 & 1 \\ 0 & -(B / M)\end{array}\right], G=$ $\left[\begin{array}{l}0 \\ 1\end{array}\right]$, and $C=\left[\begin{array}{ll}1 & 0\end{array}\right]$. 


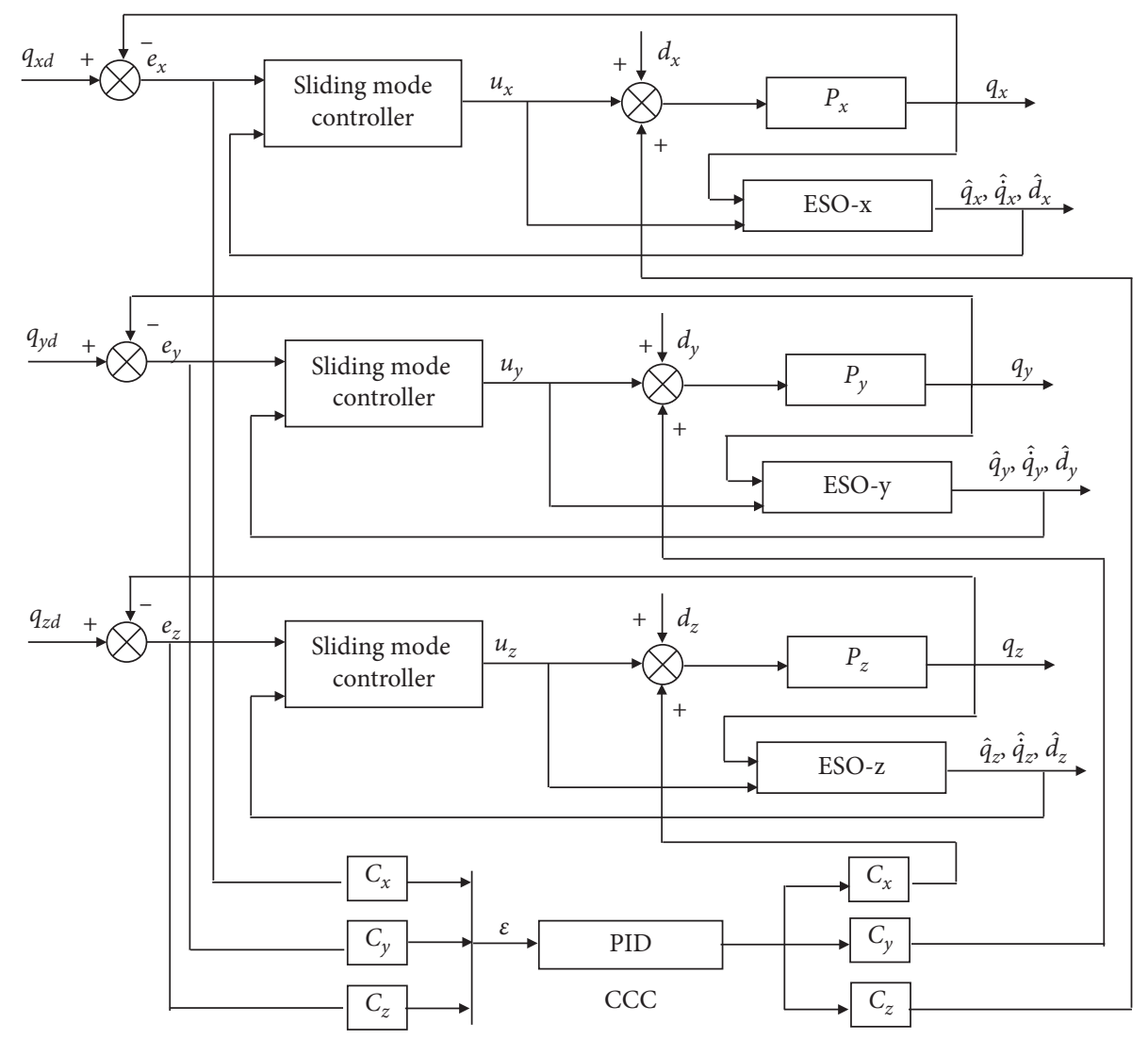

FIGURE 2: Contour error control structure diagram of the ESO SMC-based three-axis motion system.

The ESO is designed as follows:

$$
\left\{\begin{array}{l}
\dot{\hat{q}}=\widehat{\dot{q}}+\frac{\alpha_{1}}{\sigma}(q-\widehat{q}), \\
\dot{\dot{q}}=\frac{K_{f}}{M} u+\widehat{d}+\frac{\alpha_{2}}{\sigma^{2}}(q-\widehat{q}), \\
\dot{\vec{d}}=\frac{\alpha_{3}}{\sigma^{3}}(q-\widehat{q})
\end{array}\right.
$$

With this ESO, if $t \longrightarrow \infty$, obtain $\widehat{q} \longrightarrow q, \hat{\dot{q}} \longrightarrow \dot{q}$, and $\widehat{d} \longrightarrow d$, where $\widehat{q}, \widehat{\dot{q}}$, and $\widehat{d}$ are observer states. $\sigma>0$, and $\alpha_{1}$, $\alpha_{2}$, and $\alpha_{3}$ are positive real numbers, and the polynomial $s^{3}+\alpha_{1} s^{2}+\alpha_{2} s+\alpha_{3}$ satisfies the Hurwitz criterion.

Define $\mu=\left[\begin{array}{lll}\mu_{1} & \mu_{2} & \mu_{3}\end{array}\right]^{T}$.

$\mu_{1}=q-\hat{q} / \sigma^{2}, \mu_{2}=\dot{q}-\hat{\dot{q}} / \sigma$, and $\mu_{3}=d-\hat{d}$.

Due to

$$
\begin{aligned}
\sigma \dot{\mu}_{1} & =\frac{\dot{q}-\dot{\hat{q}}}{\sigma}=\frac{1}{\sigma}\left(\dot{q}-\left(\hat{\dot{q}}+\frac{\alpha 1}{\sigma}(y-\widehat{q})\right)\right)=\frac{1}{\sigma}\left(\dot{q}-\hat{\dot{q}}-\frac{\alpha 1}{\sigma}(y-\widehat{q})\right)=-\frac{\alpha 1}{\sigma^{2}}(q-\widehat{q})+\frac{1}{\sigma}(\dot{q}-\hat{\dot{q}})=-\alpha_{1} \mu_{1}+\mu_{2} \\
\sigma \dot{\mu}_{2} & =\sigma \frac{\ddot{q}-\dot{\hat{q}}}{\sigma}=\left(\frac{K_{f}}{M} u+d-\left(\frac{K_{f}}{M} u+\widehat{d}+\frac{\alpha_{2}}{\sigma^{2}}(y-\widehat{q})\right)\right)=d-\widehat{d}-\frac{\alpha_{2}}{\sigma^{2}}(y-\widehat{q}) \\
& =-\frac{\alpha_{2}}{\sigma^{2}}(q-\hat{q})+(d-\widehat{d})=-\alpha_{2} \mu_{1}+\mu_{3} \\
\sigma \dot{\mu}_{3} & =\sigma(\dot{d}-\dot{\hat{d}})=\sigma\left(\dot{d}-\frac{\alpha_{3}}{\sigma^{3}}(y-\widehat{q})\right)=\sigma \dot{d}-\frac{\alpha_{3}}{\sigma^{2}}(y-\widehat{q})=-\alpha_{3} \mu_{1}+\sigma \dot{d}
\end{aligned}
$$


The observation error state equation can be written as follows:

$$
\sigma \dot{\mu}=\bar{A} \mu+\sigma \bar{G} \dot{d}
$$

in which $\bar{A}=\left[\begin{array}{lll}-\alpha_{1} & 1 & 0 \\ -\alpha_{2} & 0 & 1 \\ -\alpha_{3} & 0 & 0\end{array}\right]$ and $\bar{G}=\left[\begin{array}{l}0 \\ 0 \\ 1\end{array}\right]$.

Characteristic equation of matrix $\mathrm{A}$ :

$$
|\lambda \mathrm{I}-\bar{A}|=\left|\begin{array}{ccc}
\lambda+\alpha_{1} & -1 & 0 \\
\alpha_{2} & \lambda & -1 \\
\alpha_{3} & 0 & \lambda
\end{array}\right|=0 .
$$

Then,

$$
\begin{aligned}
& \left(\lambda+\alpha_{1}\right) \lambda^{2}+\alpha_{3}+\alpha_{2} \lambda=0 \\
& \lambda^{3}+\alpha_{1} \lambda^{2}+\alpha_{2} \lambda+\alpha_{3}=0
\end{aligned}
$$

By selecting $\alpha_{i}(i=1,2,3)$, make $\bar{A}$ satisfy the Hurwitz criterion.

4.2. Design of the Sliding Mode Controller Based on the Extended State Observer. The sliding mode function is chosen as

$$
s=\dot{e}+c e,
$$

in which $c>0$ and $e=q-q_{d}$.

Sliding mode controller based on the extended state observer is designed as

$$
u=\left(\frac{K_{f}}{M}\right)^{-1}(-k \widehat{s}-\widehat{w}-\widehat{d}),
$$

in which $\widehat{s}=\widehat{\dot{e}}+c \widehat{e}, \quad \widehat{e}=\widehat{q}-q_{d}, \quad \widehat{\dot{e}}=\widehat{\dot{q}}-\dot{q}_{d}, \quad$ and $\widehat{\omega}=c \widehat{\dot{e}}-\ddot{q}_{d}-B / M \widehat{\dot{q}}$.

Then, the sliding mode controller can be written as

$$
u=\left(\frac{K_{f}}{M}\right)^{-1}\left(\ddot{q}_{d}+\frac{B}{M} \widehat{\dot{q}}-\hat{c} \hat{\dot{e}}-k \widehat{s}-\widehat{d}\right) .
$$

Take the Lyapunov function as

$$
V=\frac{1}{2} s^{2}
$$

Then,

$$
\begin{aligned}
\dot{V} & =s \dot{s}=s(\ddot{e}+c \dot{e})=s\left(\frac{K_{f}}{M} u+d-\frac{B}{M} \dot{q}-\ddot{q}_{d}+c \dot{e}\right) \\
& =s\left(\frac{B}{M} \widehat{\dot{q}}-c \hat{\dot{e}}-k \widehat{s}-\widehat{d}+d-\frac{B}{M} \dot{q}+c \dot{e}\right) \\
& =s\left(-\frac{B}{M} \widetilde{\dot{q}}+c \widetilde{\dot{e}}+\widetilde{d}-k \widehat{s}\right)=-k s \widehat{s}+s\left(-\frac{B}{M} \widetilde{\dot{q}}+c \widetilde{\dot{e}}+\widetilde{d}\right),
\end{aligned}
$$

in which $\widetilde{\dot{q}}=\dot{q}-\widehat{\dot{q}}, \widetilde{e}=\dot{e}-\widehat{\dot{e}}$, and $\widetilde{d}=d-\widehat{d}$.

Obviously, take the appropriate value $k$, and ensure $\dot{V} \leq 0$.
4.3. Cross-Coupled Controller. To improve the contouring accuracy, address the matching between the axes, and achieve interaxis coordinated control; the cross-coupled controller is introduced. The cross-coupled controller uses a PID control algorithm. The PID controller has a simple control algorithm and small computation amount. Furthermore, it can ensure the system real-time performance, effectively reduce the system contour error, and improve the contouring accuracy.

\section{Experiments}

To verify the effectiveness of the multiaxis contour error control approach proposed in this paper, a simulation test is conducted, in which three PMLSMs are used as the control objects of the three-axis CNC system, and the 3D saddleshape curve and screw-shape curve are selected for validation. The multiaxis CNC system is susceptible to disturbances. The three axes $x, y$, and $z$ use $d_{x}=800 \sin (\pi t)$, $d_{y}=900 \sin (2 \pi t)$, and $d_{z}=800 \sin (4 \pi t)$ to simulate the disturbance, respectively.

To reduce the impact of disturbance and improve the tracking accuracy, the sliding mode control algorithm is applied to each axis; the disturbance on each axis is observed by an ESO. Control system objects and controller parameters are shown in Table 1.

The parameters of the cross-coupled PID controller are $K p=0.01, K i=0.2$, and $K d=0.02$, respectively.

Case 1. 3D saddle-shape curve is selected for simulation. The desired curve is starting from $(2,0,0.5)$.

$$
\left\{\begin{array}{l}
x=2 \cos (2 \pi t)(\mathrm{mm}) \\
y=3 \sin (2 \pi t)(\mathrm{mm}) \\
z=0.5 \cos (4 \pi t)(\mathrm{mm}) .
\end{array}\right.
$$

Simulation results are shown in Figure 3.

Figure 3(a) shows the saddle-shape curve output in the three-dimensional space, and Figure 3(b) shows $x, y$, and $z$ three-axis's position tracking, where the solid line is the single-axis desired trajectory, and the dotted line is the actual motion trajectory. Figure 3(c) is contour error, Figure 3(d) is the perturbation on three axes and its observation, and Figure 3(e) is the varying cross-coupled gains.

It can be seen from the simulation results Figures 3(b) and $3(\mathrm{~d})$ that the uniaxial position is well tracked with the proposed single-axis sliding mode control algorithm, and the disturbances of each axis are effectively estimated with the extended state observer. Meanwhile, with the presence of the cross-coupled controller, the contour curve is well tracked. There is only a small contour error in the initial stage. This can be easily seen by comparing the input and output of the $3 \mathrm{D}$ saddle curve.

For comparison, the extended state observation for the disturbances is cancelled, and tracking error and contour error simulation results are shown in Figure 4.

It can be seen that, after canceling the extended state disturbance observation, both the single-axis tracking error and the overall contour error are significantly increased, and 
Table 1: Parameters of the 3-axis PMLSM control system.

\begin{tabular}{lccc}
\hline Parameters & $x$ axis & $y$ axis & $z$ axis \\
\hline$M$ & 5.8 & 5.8 & 1.4 \\
$K_{f}$ & 10.9 & 10.9 & 0.82 \\
$B$ & 244 & 244 & 82 \\
$c$ & 500 & 1000 & 500 \\
$k$ & 100 & 150 & 120 \\
$\alpha_{1}$ & 6 & 8 & 9 \\
$\alpha_{2}$ & 11 & 19 & 26 \\
$\alpha_{3}$ & 6 & 12 & 24 \\
$\sigma$ & 0.01 & 0.01 & 0.01 \\
\hline
\end{tabular}

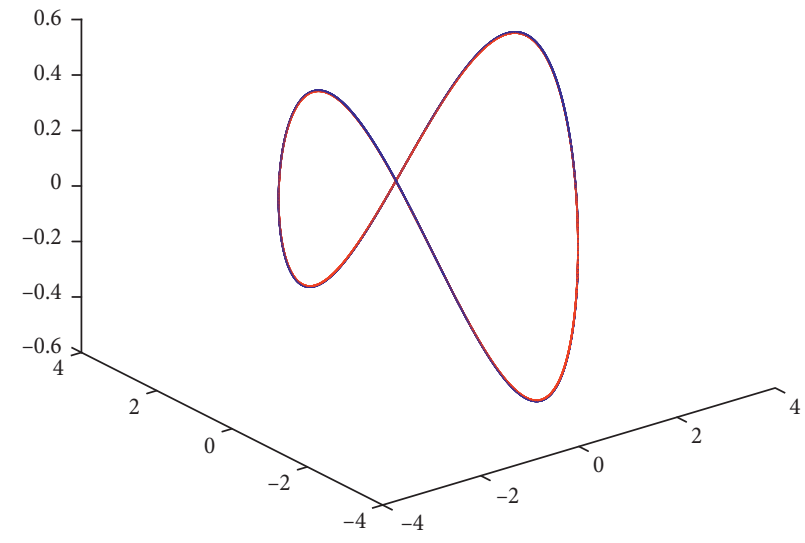

Reference contour

- Contour output

(a)

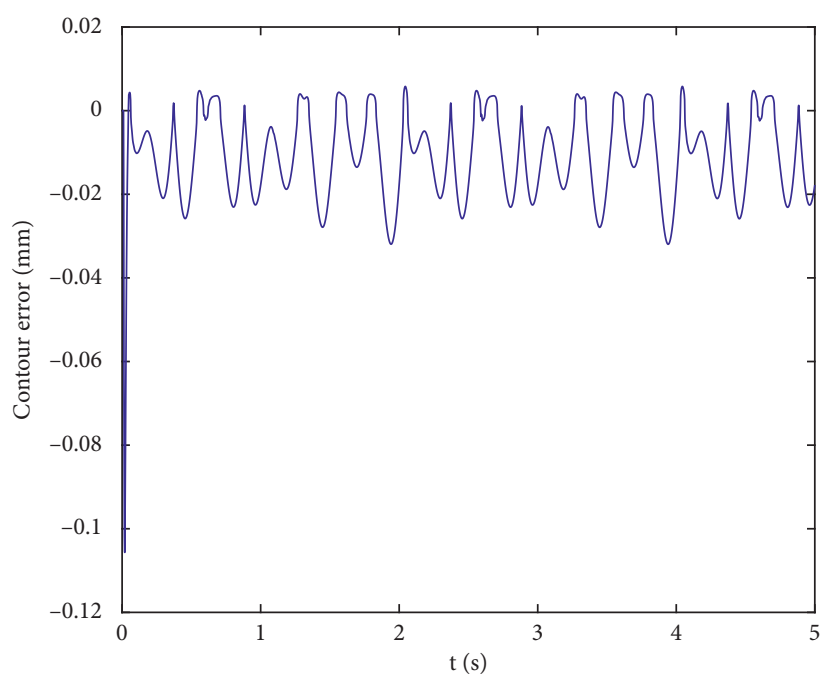

(c)
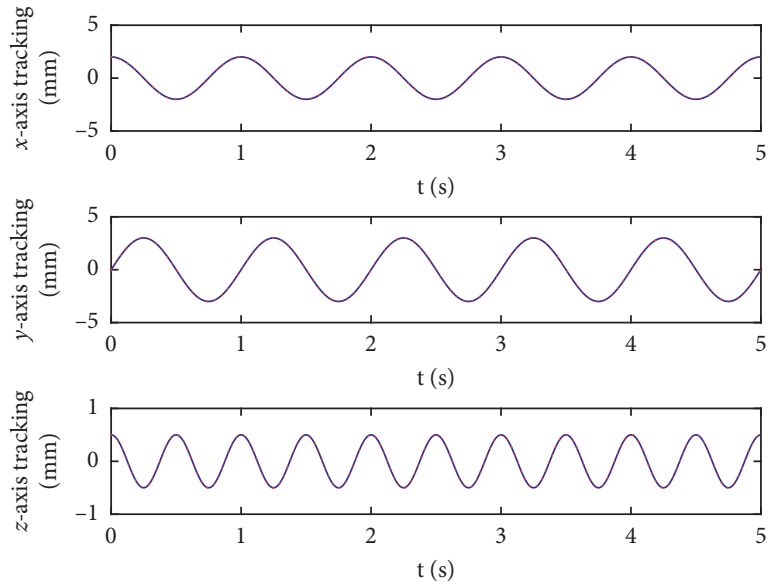

_ Ideal position Position tracking

(b)

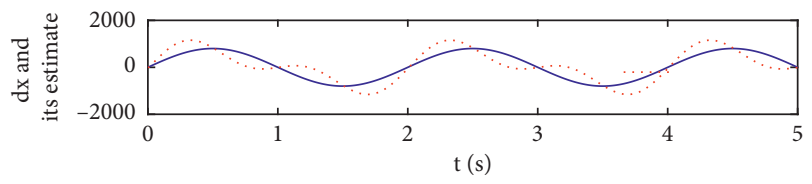

$-\mathrm{dx}$

Estimate $\mathrm{dx}$
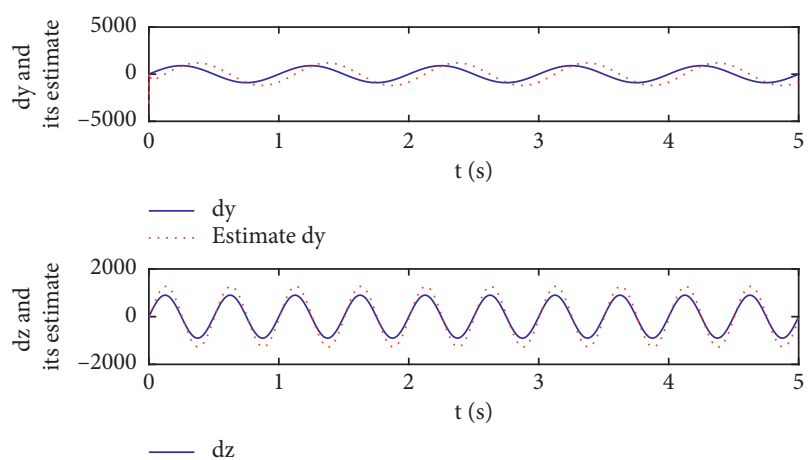

Estimate dz

(d)

FIGURE 3: Continued. 


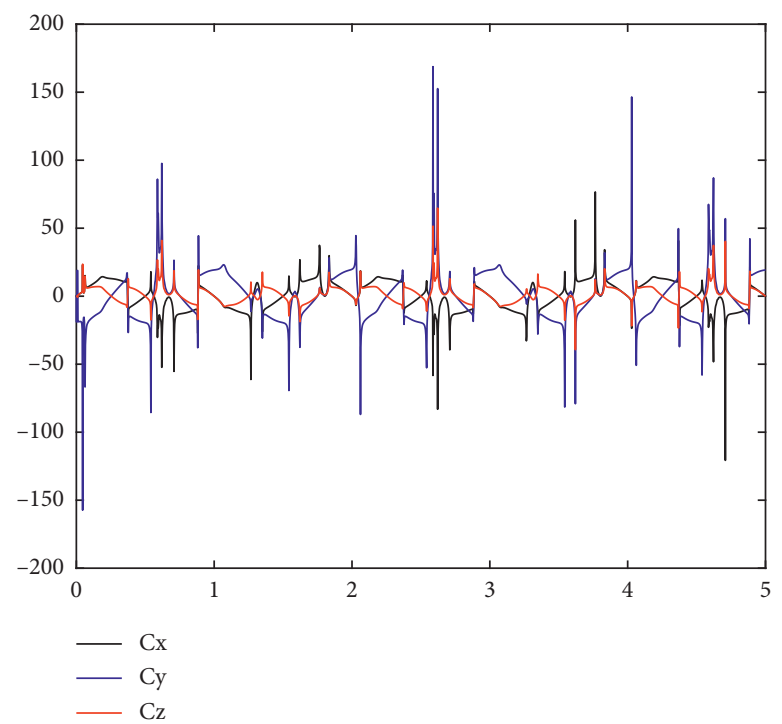

(e)

FIgURE 3: Simulation results of the 3-axis motion control system (saddle-shape curve with ESO). (a) Saddle-shape curve output. (b) Threeaxis position tracking. (c) Contour error (with ESO). (d) Triaxial perturbation and its observation. (e) $C_{x}, C_{y}$, and $C_{z}$.
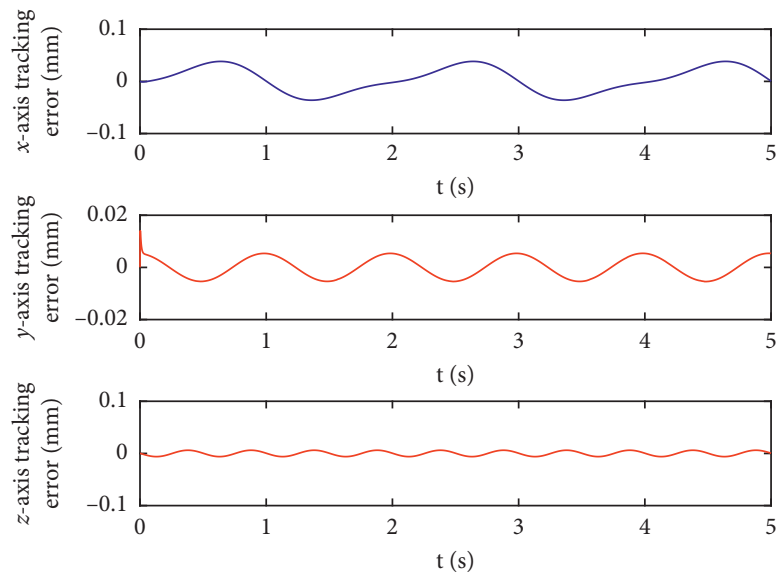

(a)

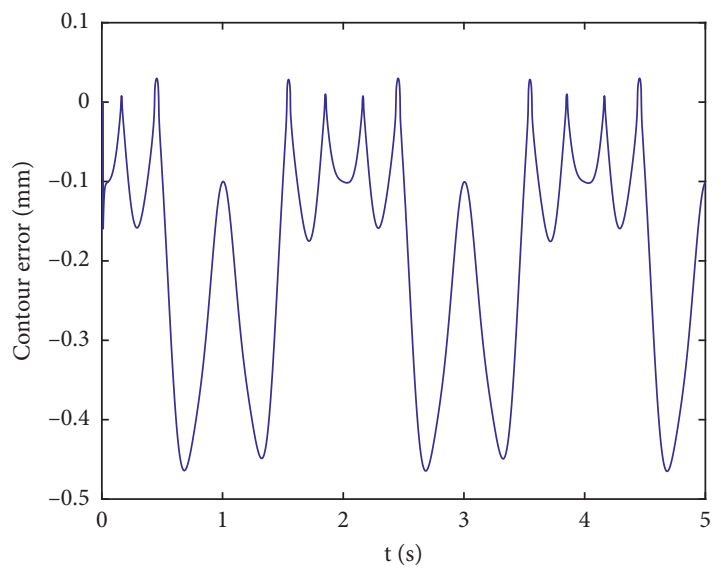

(b)

FIgURE 4: Simulation results of the 3-axis motion control system (saddle-shape curve without ESO). (a) Three-axis position tracking error. (b) Contour error (without ESO).

the control performance is degraded obviously. The comparison of specific error values is shown in Table 2 .

From the error comparison table, it can be intuitively found that, with the presence of the extended state disturbance observation, the single-axis tracking error is significantly smaller than that with the absence of the disturbance observation. The average tracking error of the $x$ axis drops to $10.19 \%$ from 0.0206 to 0.0021 ; the average tracking error of the $y$ axis drops to $42.42 \%$ from 0.0034 to 0.0014 ; and the average tracking error of the $z$ axis drops to $56.4 \%$ from 0.0039 to 0.0022 . The contour error is reduced significantly. The maximum contour error is reduced from 0.4650 to 0.1057 , the average contour error is reduced from 0.2004 to 0.012 , and the contour accuracy is improved by 16.7 times.
To further reflect the effectiveness of the control algorithm, we choose another 3D screw-shape curve for simulation analysis.

Case 2. Screw-shape curve is selected for simulation analysis. The desired curve is starting from $(3,0,0)$.

$$
\left\{\begin{array}{l}
x=3 \cos (\pi t)(\mathrm{mm}), \\
y=3 \sin (\pi t)(\mathrm{mm}), \\
z=\pi t(\mathrm{~mm})
\end{array}\right.
$$

Simulation results are shown in Figure 5.

Figure 5(a) shows screw-shape curve contour tracking, and Figure 5(b) shows $x, y$, and $z$ three-axis's position 
TABLE 2: Saddle-shape curve simulation error.

\begin{tabular}{lccc}
\hline & Simulation error $(\mathrm{mm})$ & With ESO & Without ESO \\
\hline & Maximum value- $x$ & 0.0046 & 0.0383 \\
& Mean value- $x$ & 0.0021 & 0.0206 \\
Tracking error & Maximum value- $y$ & 0.0151 & 0.0140 \\
& Mean value- $y$ & 0.0014 & 0.0034 \\
& Maximum value- $z$ & 0.0035 & 0.0061 \\
Contour error & Mean value- $z$ & 0.0022 & 0.0039 \\
& Maximum value & 0.1057 & 0.4650 \\
& Mean value & 0.0120 & 0.2004 \\
\hline
\end{tabular}

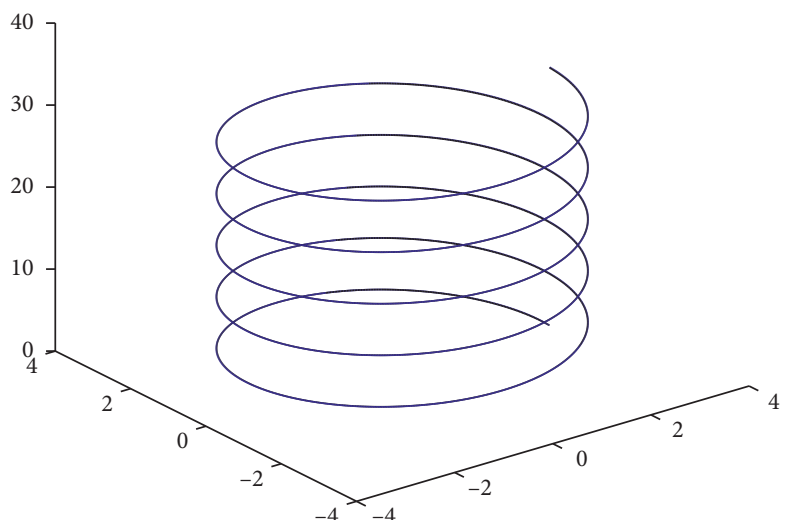

- Reference contour

_ Contour tracking

(a)

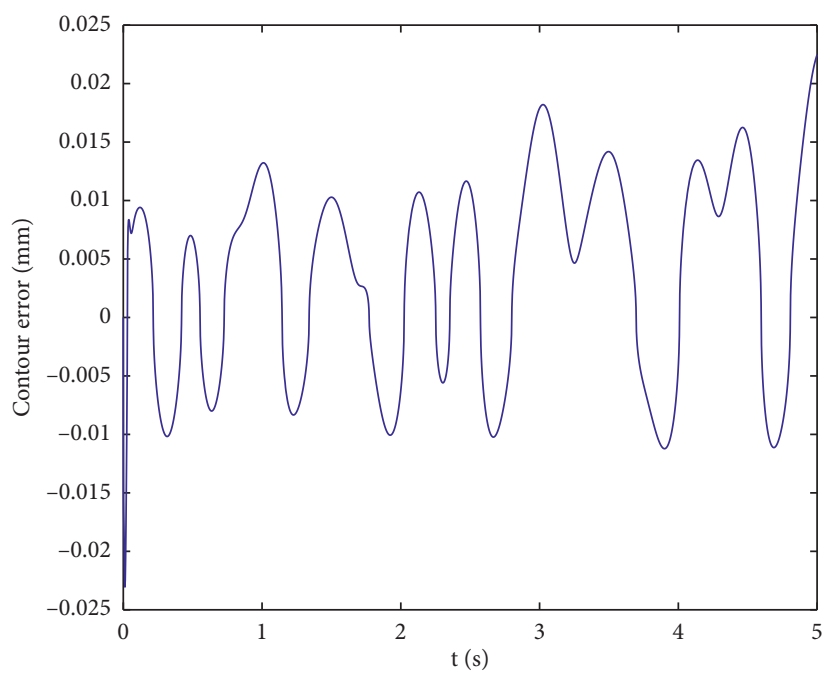

(c)
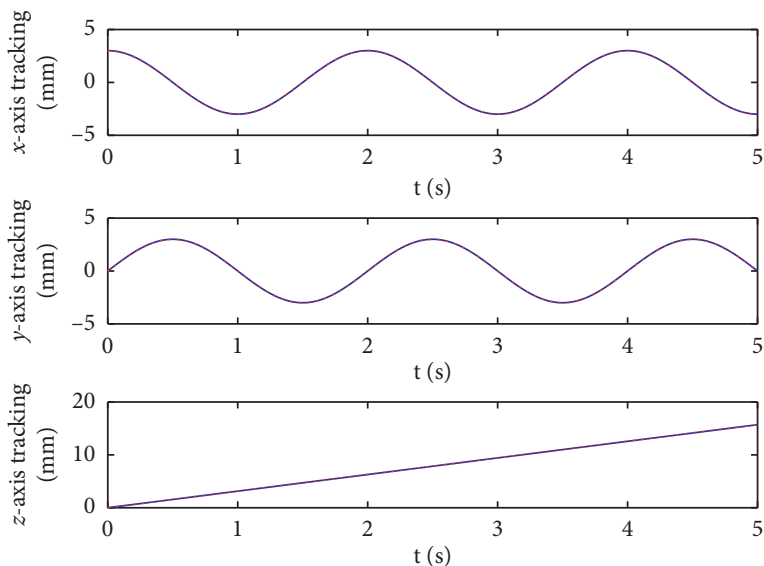

_ Ideal position Position tracking

(b)

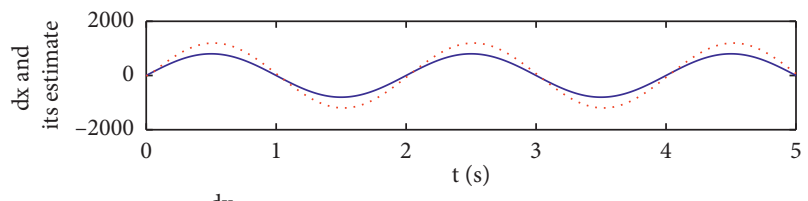

$-\mathrm{dx}$

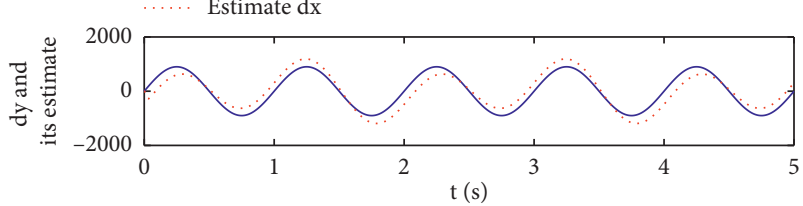

dy

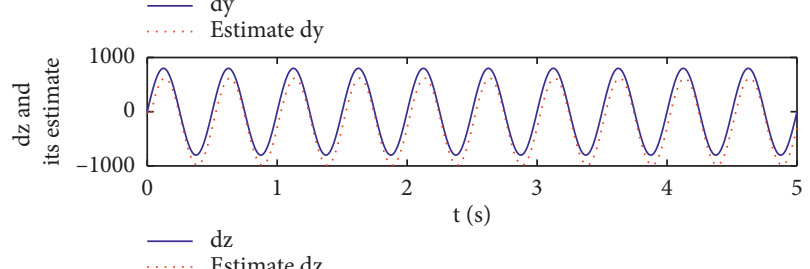

Estimate $\mathrm{dz}$

(d)

FIGURE 5: Continued. 


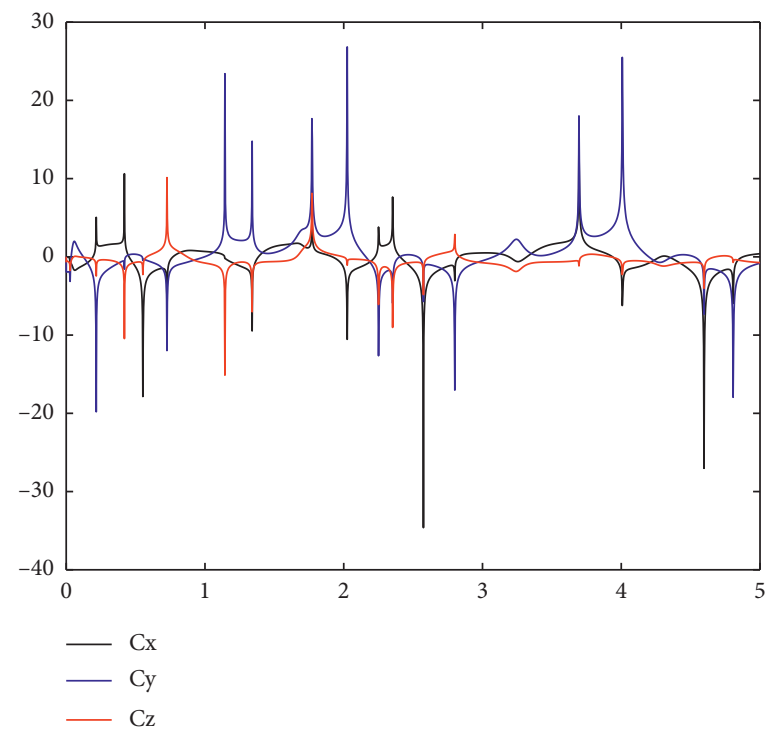

(e)

FIGURE 5: Simulation results of the 3-axis motion control system (screw-shape curve). (a) Screw-shape curve contour tracing. (b) Three-axis position tracking. (c) Contour error. (d) Triaxial perturbation and its observation. (e) $C_{x}, C_{y}$, and $C_{z}$.

TABle 3: Screw-shape curve simulation error.

\begin{tabular}{lccc}
\hline \multicolumn{2}{c}{ Simulation error $(\mathrm{mm})$} & With ESO & Without ESO \\
\hline & Maximum value- $x$ & 0.0091 & 0.0242 \\
& Mean value- $x$ & 0.0055 & 0.0157 \\
Tracking error & Maximum value- $y$ & 0.0105 & 0.070 \\
& Mean value- $y$ & 0.0026 & 0.017 \\
& Maximum value- $z$ & 0.0212 & 0.0276 \\
& Mean value- $z$ & 0.0081 & 0.0125 \\
\hline \multirow{2}{*}{ Contour error } & Maximum value & 0.0231 & 0.0523 \\
& Mean value & 0.0089 & 0.0237 \\
\hline
\end{tabular}

tracking, where the solid line is the single-axis desired trajectory, and the dotted line is the actual motion trajectory. Figure 5(c) is contour error, Figure 5(d) is the perturbation on three axes and its observation, and Figure 5(e) is the varying cross-coupled gains. The results from the screwshape curve simulation show that the proposed crosscoupled controller based on the ESO sliding mode control algorithm not only produces better single-axis tracking performance but also well tracks the contour curve. The simulation error of the screw-shape curve is shown in Table 3.

In short, the simulation results of the saddle curve and screw curve can effectively validate the effectiveness of the proposed control approach.

\section{Conclusions}

For the multiaxis linkage control system using the three-axis PMLSM servo drive, a contour error control algorithm based on the extended state disturbance observation is proposed to effectively reduce tracking error and contour error. The ESO can observe the disturbance signals to reduce the impact of the disturbances. Combine ESO with the sliding mode control algorithm to form the single-axis trajectory tracking controller with an aim of ensuring single-axis tracking accuracy and robustness. Moreover, the cross-coupled controller is applied to handle interaxis coordinated motion, make correction and compensation for the contour error of each axis, and improve dynamic performance and parameter matching of the system to ensure contour control accuracy. Next, the control algorithm is applied to the three-axis motion platform. The saddle curve contour and spiral curve contour are selected for simulation research. The results validate the effectiveness of the control algorithm.

\section{Data Availability}

The data used to support the findings of this study are included within the article.

\section{Conflicts of Interest}

The authors declare that there are no conflicts of interest.

\section{Acknowledgments}

This research was supported by the Joint Fund of Zhejiang Provincial Natural Science Foundation (Grant no. LTY20E050001) and the Natural Science Foundation of Zhejiang Province of China (Grant no.LY18F010005).

\section{References}

[1] X.-C. Xi, A.-N. Poo, and G.-S. Hong, "Tracking error-based static friction compensation for a bi-axial CNC machine," Precision Engineering, vol. 34, no. 3, pp. 480-488, 2010.

[2] Y. M. Hendrawan, K. R. Simba, and N. Uchiyama, "Iterative learning based trajectory generation for machine tool feed 
drive systems," Robotics and Computer-Integrated Manufacturing, vol. 51, pp. 230-237, 2018.

[3] D.-N. Song, J.-W. Ma, Z.-Y. Jia, F.-Z. Qin, and X.-X. Zhao, "Synergistic real-time compensation of tracking and contouring errors for precise parametric curved contour following systems," Proceedings of the Institution of Mechanical Engineers, Part C: Journal of Mechanical Engineering Science, vol. 232, no. 19, pp. 3367-3383, 2018.

[4] Y. Koren, "Cross-coupled biaxial computer control for manufacturing systems," Journal of Dynamic Systems, Measurement, and Control, vol. 102, no. 4, pp. 265-272, 1980.

[5] Y. Koren and C.-C. Lo, "Variable-gain cross-coupling controller for contouring," CIRP Annals, vol. 40, no. 1, pp. 371-374, 1991.

[6] K. H. Su and M. Y. Cheng, "Contouring accuracy improvement using cross-coupled control and position error compensator," International Journal of Machine Tools \& Manufacture, vol. 48, no. 12-13, pp. 1444-1453, 2008.

[7] J. R. Conway, C. A. Ernesto, R. T. Farouki, and M. Zhang, "Performance analysis of cross-coupled controllers for CNC machines based upon precise real-time contour error measurement," International Journal of Machine Tools and Manufacture, vol. 52, no. 1, pp. 30-39, 2012.

[8] M. Rahaman, R. Seethaler, and I. Yellowley, "A new approach to contour error control in high speed machining," International Journal of Machine Tools and Manufacture, vol. 88, pp. 42-50, 2015.

[9] J. Wu, Z. Xiong, and H. Ding, "Integral design of contour error model and control for biaxial system," International Journal of Machine Tools and Manufacture, vol. 89, pp. 159169, 2015.

[10] S. X. Wang, G. Chen, and Y. L. Cui, "Design of contour error coupling controller based on neural network friction compensation," Mathematical Problems in Engineering, 2019.

[11] D.-N. Song, Y.-G. Zhong, and J.-W. Ma, "Third-order contour-error estimation for arbitrary free-form paths in contour-following tasks," Precision Engineering, vol. 60, pp. 85-92, 2019.

[12] S. S. Yeh and P. L. Hsu, "Estimation of the contouring error vector for the cross-coupled control design," IEEE/ASME Transactions on Mechatronics, vol. 7, no. 1, pp. 0-51, 2002.

[13] H. Liu and S. Li, "Speed control for PMSM servo system Using predictive functional control and extended state observer," IEEE Transactions on Industrial Electronics, vol. 59, no. 2, pp. 1171-1183, 2011.

[14] T. Sun, J. Zhang, and Y. Pan, "Active disturbance rejection control of surface vessels using composite error updated extended state observer," Asian Journal of Control, vol. 19, no. 6, pp. 1-10, 2017. 